\title{
РОЛЬ ЦИСТАТИНА-С В РАННЕЙ ДИАГНОСТИКЕ ПРОЦЕССОВ ПОЧЕЧНОЙ ДИСФУНКЦИИ У ПОЖИЛЫХ ПАЦИЕНТОВ С АРТЕРИАЛЬНОЙ ГИПЕРТЕНЗИЕЙ И МЕТАБОЛИЧЕСКИМИ НАРУШЕНИЯМИ
}

\section{THE ROLE OF CYSTATIN-C IN EARLY DIAGNOSTICS OF RENAL DYSFUNCTION PROCESSES IN ELDERLY PATIENTS WITH ARTERIAL HYPERTENSION AND METABOLIC DISORDERS}

\section{N. Agranovich L. Albotova A. Likhacheva}

Summary. The article is devoted to the study of the level of plasma cystatin-C in elderly patients with arterial hypertension and metabolic disorders. The aim of the work is early diagnosis of chronic kidney disease. As a result of the study, it was found that the determination of plasma cystatin-C allows detecting an early preclinical decrease in renal function. The glomerular filtration rate for cystatin-C is almost twice as likely to reveal early changes in renal function as compared to the glomerular filtration rate for creatinine. This laboratory diagnostic method is more sensitive and should be used to screen for chronic kidney disease. The article can be useful for physicians, nephrologists, cardiologists.

Keywords: cystatin-C, cardiovascular diseases, kidneys, diagnostics.

\author{
Агранович Надежда Владимировна \\ Д.м.н., профессор, ФДПО ФГБОУ ВО «Ставропольский \\ государственный медицинский университет» \\ Минздрава России \\ nagranovich@mail.ru \\ Алботова Лаура Владимировна \\ Врач кардиолог, ГБУЗ «Мостовская ЦРБ», \\ Краснодарский край, пгт. Мостовской \\ doc.1.90@mail.ru \\ Лихачева Анна Петровна \\ Ассистент, ФГБОУ ВО «Ставропольский \\ государственный медицинский университет» \\ Минздрава России \\ a.anopchenko@mail.ru
}

Аннотация. Статья посвящена исследованию уровня плазменного цистатина-С у пожилых пациентов с артериальной гипертензией и метаболическими нарушениями. Цель работы ранняя диагностика хронической болезни почек. В результате исследования обнаружено, что определение плазменного цистатина-С позволяет выявлять раннее доклиническое снижение функции почек. Скорость клубочковой фильтрации по цистатину-С практически в два раза чаще выявляет ранние изменения функции почек по сравнению со скоростью клубочковой фильтрации по креатинину. Данный метод лабораторной диагностики является более чувствительным и должен применяться для скрининга хронической болезни почек. Статья может быть полезна врачам терапевтам, нефрологам, кардиологам.

Ключевые слова: цистатин-С, сердечно-сосудистые заболевания, почки, диагностика.

взаимосвязями основных хронических неинфекционных заболеваний и артериальной гипертензией. В частности, повышение артериального давления может приводить к почечной недостаточности, хронической ишемии головного мозга, в свою очередь, нарушение функции почек и психогенные факторы способствуют развитию артериальной гипертензии [5].

По данным эпидемиологических исследований, в ходе активного скрининга в регионах Российской Федерации распространенность патологии почек при артериальной гипертензии составила в среднем 43\% (1, 
3). Первичная заболеваемость гипертонической болезнью с преимущественным поражением почек на 10 тыс. взрослого населения на юге России в 1,5 раза выше, чем в среднем по РФ, а распространенность - в 1,2 раза [2].

В ряде ранее проведенных исследованиях авторами установлено, что сочетание АГ с излишней массой тела или с ожирением значительно чаще приводит к возникновению СС3, чем в случае наличия каждого из факторов по отдельности. Основными факторами, приводящими к быстрому прогрессированию патологии почек при АГ и МС, являются как сама артериальная гипертензия, так и дополнительные метаболические факторы повреждения почек: ожирение, гиперурикемия, инсулинорезистентность, нарушения липидного обмена, эндотелиальная дисфункция $[6,7,17,18]$. Учитывая, что метаболический синдром в настоящее время оценивается как пандемия 21 века, то изучение особенностей течения и влияние на АГ коморбидной патологии, в частности, метаболических нарушений, является весьма актуальным.

Ввиду отсутствия специфических симптомов ХБП трудно поддается диагностике на ранних стадиях, особенно при наличии сопутствующих заболеваний. На сегодняшний день в общеклиническом звене одним из достоверных и доступных методов определения дисфункции почек представляется показатель скорости клубочковой фильтрации (СКФ) [16], когда диагноз ХБП основывается на выявлении хронического снижения фильтрационной функции почек на фоне каких-либо структурных изменений в органе.

Вместе с тем, при использовании традиционного метода определения СКФ по концентрации креатинина крови существует вероятность получения неточных результатов, особенно у пожилых пациентов, что связано с естественным возрастным функциональным снижением скорости клубочковой фильтрации, частым приемом пациентом некоторых лекарственных средств, оказывающих влияние на скорость клубочковой фильтрации, изменение мышечной массы, и др. [19,21,22].

Это обстоятельство диктует необходимость разработки альтернативных методов определения СКФ, в частности, поиска других маркеров, по которым можно судить о наличии ранних изменений структуры и функции почек до возникновения клинических проявлений у пациентов с сопутствующими заболеваниями.

Таким подходящим маркером для определения СКФ в комплексной диагностике ХБП является цистатин-С низкомолекулярный белок с молекулярной массой 13,4 кДа, относящийся к группе ингибиторов цистеиновых протеиназ. Считают, что цистатин-С является одним из наиболее точных эндогенных маркеров СКФ, который по диагностическим характеристикам существенно превосходит креатинин. Кроме того, это высокочувствительный маркер тяжести сердечно-сосудистых событий, независимый от таких кардиомаркеров, как кардиальные тропонины, натрийуретические пептиды, С-реактивный белок и др. $[8,9,10]$. В связи с этим, цистатин-С может быть одним из предикторов развития почечной патологии на фоне сердечно-сосудистой патологии и оценки её тяжести $[18,20]$.

Комбинированное использование маркеров цистатина-С и креатинина для определения рСКФ может позволить улучшить точность оценки данного показателя у пациентов с коморбидной патологией [11].

Вместе с тем, значение цистатина-С для прогнозирования развития ХБП у больных старшей возрастной группы с АГ и метаболическими нарушениями в настоящее время остается малоизученным, что требует необходимости дальнейшие исследования.

Цель исследования:

1. Оценить прогностическое значение уровня цистатина-С и его взаимосвязь с риском развития ХБП у пожилых больных с АГ и АГ с метаболическими нарушениями.

2. Оценить влияние метаболических нарушений на прогрессирование ХПН у пожилых больных c AГ.

\section{Материалы и методы исследования}

Исследование проведено на клинических базах кафедры поликлинической терапии Ставропольского медицинского университета.

В исследовании приняли участие 348 пациентов, старше 55 лет, страдающих гипертонической болезнью. Во всех случаях диагноз заболевания выставлен согласно клиническим рекомендациям диагностики и лечения АГ (Москва, 2013 г.) и подтвержден данными инструментальных и лабораторных исследований.

Все пациенты были разделены на две группы. В первую группу вошли 163 пациента с диагностированной гипертонической болезнью и не имеющих метаболического синдрома, во вторую группу - 185 человек, с АГ и наличием метаболического синдрома. Средний возраст пациентов в первой группе составил 65,2 20,2 , во второй 64,8 0,2 года. Для включения в исследование всеми больным было подписано «Информированное согласие пациента».

К критериям включения в исследование относились: 
Таблица 1. Характеристика обследованных пациентов по параметрам физического развития

\begin{tabular}{|c|c|c|c|c|c|c|}
\hline \multirow{3}{*}{ Показатель } & \multicolumn{6}{|c|}{ Артериальная гипертензия } \\
\hline & \multicolumn{2}{|c|}{ Мужчины $(\mathrm{M} \pm \mathrm{m})$} & \multirow{2}{*}{$t, p$} & \multicolumn{2}{|c|}{ Женщины (M \pm m) } & \multirow{2}{*}{$t, p$} \\
\hline & Без MC & $\mathrm{CMC}$ & & Без MC & C MC & \\
\hline Масса тела, кг & $75,81 \pm 0,48$ & $80,76 \pm 0,54$ & $\begin{array}{l}t>4, \\
\rho<0,001\end{array}$ & $71,82 \pm 0,42$ & $78,43 \pm 0,49$ & $\begin{array}{l}t>4, \\
\rho<0,001\end{array}$ \\
\hline PoCT, CM & $176,9 \pm 0,46$ & $177,2 \pm 0,5$ & $\begin{array}{l}t<2, \\
\rho>0,05\end{array}$ & $166,9 \pm 0,6$ & $165,4 \pm 0,5$ & $t<2, \rho>0,05$ \\
\hline ИМТ (Kг/M²) & $25,28 \pm 0,44$ & $26,74 \pm 0,52$ & $\begin{array}{l}t>2, \\
\rho<0,05\end{array}$ & $25,86 \pm 0,53$ & $28,13 \pm 0,45$ & $t>3, \rho<0,01$ \\
\hline OT, CM & $84,0 \pm 0,7$ & $86,9 \pm 0,8$ & $\begin{array}{l}t>2, \\
\rho<0,05\end{array}$ & $82,4 \pm 1,0$ & $91,7 \pm 0,9$ & $\begin{array}{l}>4, \\
\rho<0,001\end{array}$ \\
\hline OБ, сM & $99,0 \pm 0,8$ & $99,7 \pm 1,0$ & $\begin{array}{l}t<2, \\
\rho>0,05\end{array}$ & $91,7 \pm 0,9$ & $101,8 \pm 1,2$ & $\begin{array}{l}t>4, \\
\rho<0,001\end{array}$ \\
\hline ОТ/OБ & $0,87 \pm 0,5$ & $0,88 \pm 0,9$ & $\begin{array}{l}t<2, \\
\rho>0,05\end{array}$ & $0,84 \pm 0,8$ & $0,92 \pm 0,95$ & $t<2, \rho>0,05$ \\
\hline
\end{tabular}

1. пациенты с АГ I-III степеней, согласно клиническим рекомендациям диагностики и лечения АГ (Москва, 2013 г.);

2. пациенты с метаболическим синдромом, который включает: абдоминально-висцеральное ожирение, инсулинорезистентность, гиперинсулинемию, дислипидемию, нарушение толерантности к глюкозе;

К критериям исключения относились:

1. симптоматические АГ;

2. наличие тяжелых соматических заболеваний;

3. проявления острой почечной недостаточности;

4. нарушения ритма сердца, требующие постоянной антиаритмической терапии;

Обследование больных включало: сбор жалоб, анамнестических данных, общий осмотр. Всем пациентам выполнялась антропометрия с расчетом ИМТ.

Всем больным проводили: общий анализ крови и мочи, биохимические исследования (холестерин, триглицериды, ЛПНП, ЛПВП, креатинин, мочевина, общий белок и его фракции, СРБ, фибриноген, ПТИ, цистатин С). Электрокардиография в 12 отведениях и ЭхоКГ проводилось при посещении пациентом поликлиники вначале исследования.

Статистическую обработку полученных данных проводили с использованием стандартного пакета программы Statistica 10 (StatSoft Inc, 2011, США). Качественные переменные описывались абсолютными и относительными (\%) частотами, для количественных переменных определяли среднее арифметическое и стандартную ошибку средней (M $\pm \mathrm{m})$. При сравнении двух групп по качественным признакам использовали критерий хи-квадрат (х2). Сравнение двух связанных между собой групп по количественным признакам осуществляли непараметрическим методом с использованием теста согласованных пар Вилкоксона. Сравнение двух не связанных между собой групп по количественным признакам осуществляли непараметрическим методом с использованием U-критерия Манна-Уитни. Для всех видов анализа статистически значимыми считали различия при $\mathrm{p}<0,05$.

\section{Результаты исслеАования}

При изучении факторов риска отмечено, что абдоминальное ожирение встречалось у 39\% обследованных пациентов, варианты атерогенной дислипидемий у $29,85 \%$, умеренное употребление алкоголя 7,46\%, курили $-21 \%$.

ИМТ > 28 кг/м² имели 6,2\% (5 чел.) мужчин не имевших метаболических нарушений и 8,4\% (8 чел.) женщин, а в группе сочетавших АГ с метаболическим синдромом - 19,8\% (16 чел.) и 38,9\% (31 чел.) соответственно. Распределение жировой ткани у всех пациентов оценивалось по абдоминальному типу, который характеризуется величиной ОТ/ОБ более 0,8 (Табл. 1).

Уровень систолического артериального давления был достоверно выше у больных с выраженными метаболическими нарушениями, тогда как существенные различия в уровне диастолического давления отмечались лишь у пациентов со второй стадией АГ и метаболическим синдромом (Табл. 2).

В группе больных с АГ и МС чаще выявлялись поражения органов-мишеней сосудов глазного дна (53,73\%) и гипертрофии левого желудочка (52,34\%).

Среднее значение креатинина крови у пациентов с ГБ и метаболическими нарушениями оказалось равным 
Таблица 2. Распределение пациентов по уровню артериального давления в зависимости от степени гипертонической болезни и наличию метаболического синдрома

\begin{tabular}{|c|c|c|c|c|c|c|}
\hline \multirow{2}{*}{$\begin{array}{l}\text { Степень } \\
\text { гипертонической } \\
\text { болезни }\end{array}$} & \multicolumn{2}{|c|}{ САД, мм.рт.ст. (M \pm m) } & \multirow[b]{2}{*}{$t, p$} & \multicolumn{2}{|c|}{ ДАД, мм.рт.ст. (M \pm m) } & \multirow[b]{2}{*}{$t, p$} \\
\hline & АГ без МС & АГ с MC & & АГ без MC & АГ С MC & \\
\hline Первая & $134.57 \pm 1,08$ & $141.25 \pm 1,25$ & $t>3, p<0,01$ & $84.14 \pm 1,25$ & $85.54 \pm 1,42$ & $t<2, p>0,05$ \\
\hline Вторая & $140.35 \pm 0,85$ & $147.62 \pm 1,09$ & $t>4, p<0,001$ & $87.54 \pm 0,86$ & $90.55 \pm 0,78$ & $t>2, p<0,05$ \\
\hline Третья & $157.19 \pm 1,05$ & $164.20 \pm 0,98$ & $t>4, p<0,001$ & $95.35 \pm \mathbf{0 , 8 9}$ & $96.80 \pm 0,79$ & $t<2, p>0,05$ \\
\hline
\end{tabular}

Таблица 3. Средняя скорость клубочковой фильтрации в мл/мин/1,73 м² у пациентов с АГ и АГ с метаболическим синдромом

\begin{tabular}{|c|c|c|c|}
\hline \multirow{2}{*}{$\begin{array}{l}\text { Степень гипертонической } \\
\text { болезни }\end{array}$} & \multicolumn{2}{|c|}{ Скорость клубочковой фильтрации (М \pm m) } & \multirow{2}{*}{$t, p$} \\
\hline & АГ без МС & АГ с MC & \\
\hline Первая & $98,17 \pm 0,96$ & $96,46 \pm 0,91$ & $t<2, p>0,05$ \\
\hline Вторая & $93,06 \pm 0,66$ & $91,50 \pm 0,56$ & $t<2, p>0,05$ \\
\hline Третья & $89,44 \pm 0,55$ & $87,72 \pm 0,49$ & $t>2, p<0,05$ \\
\hline Итого & $92,22 \pm 0,48$ & $90,99 \pm 0,42$ & $t<2, p>0,05$ \\
\hline
\end{tabular}

Таблица 4. Средний уровень цистатина-С у пожилых пациентов в 1 и 2 группах (в мг/л)

\begin{tabular}{|l|l|l|l|}
\hline Степень тяжести течения АГ & Пациенты с АГ & Пациенты с АГ + МС & $\mathbf{t}, \mathbf{p}$ \\
\hline 1 степень & $0,73 \pm 0,03$ & $0,94 \pm 0,04$ & $t>4, \rho<0,001$ \\
\hline 2 степень & $0,82 \pm 0,02$ & $1,22 \pm 0,03$ & $t>4, \rho<0,001$ \\
\hline Встепень & $0,84 \pm 0,02$ & $1,29 \pm 0,03$ & $t>4, \rho<0,001$ \\
\hline Всего & $0,81 \pm 0,01 \mathrm{mг} / л$ & $1,21 \pm 0,02$ & $t>4, \rho<0,001$ \\
\hline
\end{tabular}

78,92 $\pm 0,83$, в группе больных АГ без метаболического синдрома - 75,74 $\pm 0,77$ мкмоль/л, что достоверно ниже, чем в основной группе, но оба показателя находятся в пределах референтных интервалов уровня креатинина сыворотки крови. Рассчет скорости клубочковой фильтрации по формуле CKD-EPI показал, что средняя CKФ у 35,2 $\pm 3,5 \%$ пациентов в обеих группах имеют показатели ниже 90 мл/мин/1,73м². У больных с АГ и метаболическим синдромом существенно не отличалась от больных сравниваемой группы с АГ без МС, лишь при третьей степени АГ с метаболическим синдромом наблюдается статистически достоверное снижение СКФ. (Табл. 3).

У значительной части пациентов, имеющих СКФ <90 мл/мин/1,73м², уровень креатинина в сыворотке крови находится в пределах нормы, что предполагает существование так называемой «креатинин - слепой» области СКФ (40-70 мл/мин/1,73м²). Что еще раз обосновывает применение альтернативных методов диагностики почечной дисфункции.

Проведенное исследование уровня цистатина-С в обеих группах показало достоверные изменения (Табл. 4).

Нужно отметить, что у лиц с АГ и метаболическими нарушениями показатели оказались достоверно выше (38,9\%), чем у пациентов с АГ не имеющих метаболиче- ских нарушений (3,4\%). Концентрация цистатина-С в сыворотке крови у больных АГ и МС варьировала от 900 до $1600 н г / м л$. СКФ, рассчитанная по цистатину-C (CKDEPI cys $=100 /$ цистатин-C -14 (мл/мин/1,73 м2) была достоверно ниже в группе пациентов имеющих метаболический синдром. (Табл. 5).

Проведенный анализ позволил выявить ранние нарушения СКФ при АГ у пациентов пожилого возраста. Причем при первой и второй степени АГ с метаболическими нарушениями СКФ достоверно ниже, чем у тех, кто не имел метаболических расстройств $(t>2, \rho<0,05)$. Сопоставляя среднюю СКФ, рассчитанной по цистатину-С и по креатинину удается выявить незначительные нарушения функции почек в 2,3 раза чаще. Особенно большое значение это имеет для больных АГ с первой и второй степенью тяжести, поскольку своевременная коррекция назначенного лечения позволяет предупредить развитие ХБП и снизит риск развития ОПП.

Проведенный сравнительный анализ уровня цистатина-С и степени тяжести АГ показал, что при АГ 1 степени умеренное снижение функции почек было выявлено у 1 больного, тогда как в группе пациентов с артериальной гипертонией и метаболическим синдромом у 12. (Табл. 6).

Так же нами установлен тот факт, что у пациентов с АГ и метаболическими нарушениями прогрессиро- 
Таблица 5. Средняя скорость клубочковой фильтрации по цистатину-С (CKD-EPI суs) у пациентов с АГ и с АГ с метаболическими нарушениями (мл/мин/1,73м²).

\begin{tabular}{|c|c|c|c|}
\hline \multirow{2}{*}{$\begin{array}{l}\text { Степень артериальной } \\
\text { гипертензии }\end{array}$} & \multicolumn{3}{|c|}{ Скорость клубочковой фильтрации (M $\pm \mathrm{m})$} \\
\hline & АГ без MC & АГ с MC & $t / p$ \\
\hline Первая & $93,86 \pm 1,73$ & $87,11 \pm 2,21$ & $t>2, \rho<0,05$ \\
\hline Вторая & $86,24 \pm 1,46$ & $80,44 \pm 1,19$ & $t>2, \rho<0,05$ \\
\hline Третья & $71,16 \pm 1,7$ & $71,93 \pm 1,43$ & $t<2, \rho>0,05$ \\
\hline Итого & $81,9 \pm 1,18$ & $78,00 \pm 0,94$ & $t>2, \rho<0,05$ \\
\hline
\end{tabular}

Таблица 6. Распределение больных в зависимости от скорости клубочковой фильтрации, тяжести течения артериальной гипертонии и стадии почечной недостаточности

\begin{tabular}{|c|c|c|c|c|c|}
\hline \multirow{2}{*}{ Стадия } & \multirow{2}{*}{$\begin{array}{l}\text { Уровень СКФ по цистатину-С } \\
\text { (мл/мин/1,73 м2) }\end{array}$} & \multirow{2}{*}{$(\mathrm{P} \pm \mathrm{m} \%)$} & \multicolumn{3}{|c|}{ Степени тяжести АГ } \\
\hline & & & 1степень & 2степень & Зстепень \\
\hline \multirow{3}{*}{ C1 } & \multirow{3}{*}{ >90(оптимальная) } & $\mathrm{A} \Gamma$ & $62,86 \pm 8,17$ & $38,03 \pm 5,76$ & $1,75 \pm 1,74$ \\
\hline & & $\mathrm{A} \Gamma+\mathrm{MC}$ & $35,71 \pm 9,05$ & $23,2 \pm 4,7$ & $6,67 \pm 2,88$ \\
\hline & & $p$ & $<0,05$ & $<0,05$ & $>0,05$ \\
\hline \multirow{3}{*}{ C2 } & \multirow{3}{*}{$\begin{array}{l}\text { 60-89 (Незначительно } \\
\text { сниженная) }\end{array}$} & $\mathrm{A} \Gamma$ & $37,14 \pm 8,17$ & $60,56 \pm 5,8$ & $73,68 \pm 5,83$ \\
\hline & & $\mathrm{A} \Gamma+\mathrm{MC}$ & $64,3 \pm 9,1$ & $67,1 \pm 5,2$ & $73,33 \pm 5,1$ \\
\hline & & $p$ & $<0,05$ & $>0,05$ & $>0,05$ \\
\hline \multirow{2}{*}{$\mathrm{C} 3 \mathrm{a}$} & \multirow{2}{*}{ 45-59 (Умеренно сниженная) } & $\mathrm{A} \Gamma$ & $0,0 \pm 0,0$ & $1,41 \pm 1,40$ & $19,30 \pm 5,23$ \\
\hline & & $\mathrm{A} \Gamma+\mathrm{MC}$ & $0,0 \pm 0,0$ & $1,2 \pm 1,2$ & $17,3 \pm 4,4$ \\
\hline \multirow{2}{*}{ C36 } & \multirow{2}{*}{$\begin{array}{l}\text { 30-44 (Существенно } \\
\text { сниженная) }\end{array}$} & $\mathrm{A} \Gamma$ & $0,0 \pm 0,0$ & $0,0 \pm 0,0$ & $5,26 \pm 2,96$ \\
\hline & & $\mathrm{A} \Gamma+\mathrm{MC}$ & $0,0 \pm 0,0$ & $0,0 \pm 0,0$ & $2,7 \pm 1,9$ \\
\hline
\end{tabular}

вание хронической болезни почек идет быстрее, доля пациентов со сниженным СКФ выше, чем у пациентов страдающих АГ без метаболического синдрома. Оптимальная функция почек при первой степени АГ у пациентов с метаболическим синдромом встречалась 1,8 раз реже, чем у их сверстников без метаболического синдрома, а доля лиц с незначительно сниженной функцией почек оказалась достоверно выше. Аналогичные закономерности отмечаются и в группе больных со второй степенью тяжести АГ. Статистически значимых различий при третьей степени тяжести АГ выявлено не было.

\section{Обсужление результатов \\ исслеАования и выво}

Таким образом, проведенные исследования показали, что одним из достоверных маркеров раннего выявления и прогнозирования нефропатии может стать цистатин-С., особенно у лиц пожилого и старческого возраста, т.к. его концентрация не зависит от мышечной массы, возраста, пола. Предпочтительность оценки СКФ по уровню креатинина и/или цистатина-С у разных категории больных продолжает обсуждаться. Вместе с тем, диагностическая ценность цистатина-С для определения нарушенной СКФ, проанализированная в нашем исследовании у 348 больных показало превосходство по сравнению с креатинином, особенно у пациентов АГ В сочетаний с метаболическими нарушениями. У паци- ентов, с нормальной СКФ внепочечные факторы в большей степени влияют на сывороточную концентрацию креатинина, чем цистатин-С, поэтому креатинин нечувствителен к выявлению незначительного снижения СКФ.

Согласно нашим данным, концентрация цистатина-С в сыворотке крови у больных с АГ и МС была достоверно выше, чем в группе контроля и находилась в пределах от 900 до 1600 нг/мл (в контрольной группе - от 500 до $900 н г / м л)$.

В настоящем исследовании у пациентов с АГ и метаболическими нарушениями обнаружена обратная корреляция цистатина-С со СКФ: концентрация цистатина-С увеличивалась по мере снижения СКФ и позволила выявлять раннее доклиническое снижение функции почек. Скорость клубочковой фильтрации по цистатину-С практически в два раза чаще выявляет ранние изменения функции почек по сравнению со скоростью клубочковой фильтрации по креатинину, что позволяет констатировать этот метод лабораторной диагностики является более чувствительным и должен применяться для скрининга ХБП. В нашем исследовании доля пациентов с выявленным повышенным содержанием цистатина-С среди лиц с метаболическими нарушениями значительно выше, что позволяет использовать их как маркеры субклинических ренальных осложнений при гипертонической болезни сочетающейся с метаболическим синдромом. 
Определение цистатина-С у больных пожилого возраста, страдающих АГ и МС, помогает ранней диагностике почечной дисфункций, что позволяет поводить своевременное лечение, оказывая позитивное влияние на течение основного заболевания и улучшить качество жизни больных.

\section{ЛИТЕРАТУРА}

1. Агранович Н.В., Анопченко А. С., Пустовей Д. В., Пилипович Л. А. Ранняя диагностика поражения почек при артериальной гипертензии у пожилых пациентов // Международный журнал прикладных и фундаментальных исследований. — 2014. — № 11-5.—C. 817-819;

2. Агранович Н.В., Пустовей Д. В., Алботова Л.В Анализ взаимосвязи развития хронической болезни почек у пациентов старших возрастных групп с сердечно-сосудистой патологией. Задачи амбулаторного звена в ранней диагностике и профилактике// Современные проблемы науки и образования.2015. 一 № 5 .

3. Агранович Н. В. Качественная диагностика — ключ кэффективному лечению хронических воспалительных заболеваний почек в условиях поликлиники/ Агранович Н. В., Пилипович Л. А., Алботова Л. В., Кнышова С. А. // Актуальные вопросы современной медицины: материалы IX межрегиональной научно-практической конференции для врачей первичного звена здравоохранения Северо-Кавказского федерального округа «Качество жизни лиц пожилого и старческого возраста — зеркало здоровья населения» / Под общей ред. В. И. Кошель.—Ставрополь - Владикавказ. Изд-во СТГМУ, 2017. — С. 64-67.

4. Дедов И. И. Сахарный диабет и артериальная гипертензия / И. И. Дедов, М. В. Шестакова. — Москва. — 2009. — 342 с.

5. Кобалава Ж.Д., Виллевальде С. В., Моисеев В. С. Сердечно-сосудистые заболевания и функциональное состояние почек // Рос. кардиол. журн.— 2013.— № 4 (102). - С. 33-37.

6. Мустафаева А.Г., Алиева Т.Т. Изучение некоторых биохимических показателей, ассоциированных с патологией сосудистой системы при метаболическом синдроме //Актуальні проблеми сучасної медицини: Вісник української медичної стоматологічної академії. - 2018. - Т. 18, № 1 (61). - С. 94-98.

7. Устинова М.А., Батюшин М.М. Анализ влияния маркеров почечного повреждения при нарушении углеводного обмена на риск появления неселективной протеинурии. - // Клиническая нефрология. - 2017. -№ 4. - С. 51-55.

8. Abdel-Salam M., Ibrahim S., Pessar S. A., Al-Morsy E. The relationship between serum homocysteine and highly sensitive C-reactive protein levels in children on regular hemodialysi // Saudi J. Kidney Dis. Transpl.—2017.—Vol. 28 (3) - P. 483-490.

9. Bevc S., Ekart R., Hojs R. Cystatin C — a marker of kidneyfunction and predictor of cardiovascular diseaseand mortality // Acta Med Biotechn. — 2014. — Vol.7.— P. 9-15.

10. Chen S., Hsu W. Y., Lin Y. N. et al. Incidence and risk of major adverse cardiovascular events in middle-aged patients with chronic kidney disease: a population-based cohort study // Int. Urol. Nephrol. — 2019.—Vol. 51 (7) - P. 1219-1227.

11. Deng Y., Wang L., Hou Y. et al. The influence of glycemic status on the performance of cystatin C for acute kidney injury detection in the critically ill // Ren. Fail.2019. - Vol. 41 (1) - P. 139-149.

12. Gansevoort R.T., Correa-Rotter R., Hemmelgarn B. R.et al. Chronic kidney diseaseand cardiovascular risk: epidemiology, mechanisms, and prevention //Lancet.2013.-Vol.382.-P. 339-352.

13. Garasto S., Fusco S., Corica F. et al. Estimating glomerular filtration rate in older people // Biomed. Res. Int. — 2014. — Vol.2014. — P. 916542.

14. Kim S., Hwang S., Jang H. R. et al. Creatinine- and cystatin C-based estimated glomerular filtration rate slopes for the prediction of kidney outcome: a comparative retrospective study // BMC Nephrol. - 2019._-Vol. 20 (1) - P. 214.

15. Lamb E.J., Stevens P.E. Estimating and measuring glomerular filtration rate: methods of measurement and markers for estimation // Curr. Opin. Nephrol. Hypertens. - 2014.-Vol.23.-P. 258-266.

16. Levey A.S., Becker C., Inker L. A. Glomerular filtration rate and albuminuria for detection and staging of acute and chronic kidney disease in adults: a systematic review // JAMA. - 2015.—Vol.313.—P. 837-846.

17. Mao Q., Zhao N., Wang Y. et al. Association of Cystatin C with Metabolic Syndrome and Its Prognostic Performance in Non-ST-Segment Elevation Acute Coronary Syndrome with Preserved Renal Function // Biomed. Res. Int. — 2019._ Vol. 2019-8541402.

18. Osaki T., Satoh M., Tanaka F. et al. The Value of a Cystatin C-based Estimated Glomerular Filtration Rate for Cardiovascular Assessment in a General Japanese Population: Results from the Iwate Tohoku Medical Megabank Project // J. Epidemiol.— 2019._- May 25. doi: 10.2188/jea.JE20180274. [Epub ahead of print].

19. Rowe C., Sitch A. J., Barratt J. et al. Biological variation of measured and estimated glomerular filtration rate in patients with chronic kidney disease // Kidney Int. 2019. - Vol. 96 (2) - P. 429-435.

20. Shlipak M.G., Mattes M. D., Peralta C. A. Update on cystatinC: incorporation into clinical practice // Am. J. KidneyDis. — 2013. — Vol.62. — P. 595-603.

21. Shlipak M.G., Matsushita K. Cystatin C versus creatinine in determiningrisk based on kidney function //N Engl J Med. — 2013.—Vol.369.—P. 932-943.

22. Zonoozi S., Ramsay S. E., Papacosta 0. et al. Chronic kidney disease, cardiovascular risk markers and total mortality in older men: cystatin Cversus creatinine // J. Epidemiol. Community Health. - 2019. - Vol. 73 (7) - P. 645-651.

(c) Агранович Надежда Владимировна ( nagranovich@mail.ru ), 